\title{
Use of radiotherapy in patients with palliative double bypass for locally advanced pancreatic adenocarcinoma
}

\author{
Juan Glinka, MD1, Federico Diaz, MD², Augusto Alva, MD², Oscar Mazza, MD', \\ Rodrigo Sanchez Claria, MD¹, Victoria Ardiles, MD', Eduardo de Santibañes, MD, PhD', \\ Juan Pekolj, MD, PhD ${ }^{1}$, Martín de Santibañes, MD \\ 'Department of General Surgery, Hepato-Bilio-Pancreatic Unit, Hospital Italiano de Buenos Aires, Buenos Aires; \\ ${ }^{2}$ Department of Radiation Oncology, Hospital Italiano de Buenos Aires, Buenos Aires, Argentina
}

Purpose: Pancreatic cancer (PC) has not changed overall survival in recent years despite therapeutic efforts. Surgery with curative intent has shown the best long-term oncological results. However, 80\%-85\% of patients with these tumors are unresectable at the time of diagnosis. In those patients, first therapeutic attempts are minimally invasive or surgical procedures to alleviate symptoms. The addition of radiotherapy (RT) to standard chemotherapy, ergo chemoradiation, in patients with locally advanced pancreatic cancer (LAPC) is still controversial. The study aims to compare outcomes in patients with a double bypass surgery due to LAPC treated or not with RT.

Materials and Methods: A retrospective cohort study of patients with double bypass for LAPC were registered and divided into two groups: treated or not with postoperative RT. Baseline characteristics, postoperative complications, those related to RT and their relation to the main event (mortality) were compared.

Results: Seventy-four patients were included. Surgical complications between the groups did not offer significant differences. Complications related to RT were mostly mild, and $86 \%$ of patients completed the treatment. Overall survival at 1 and 2 years for patients in the exposed group was $64 \%$ and $35 \%$ vs. $50 \%$ and $28 \%$ in the non-exposed group, respectively ( $p=0.11$; power $72 \%$; hazard ratio $=0.53 ; 95 \%$ confidence interval, $0.24-1.18$ ).

Conclusion: We observed a tendency for survival improvement in patients with postoperative RT. However, we've not had enough power to demonstrate this difference, possibly due to the small sample size. It is indispensable to develop randomized and prospective trials to guide more specific treatment lines in this patients.

Keywords: Pancreatic cancer, Radiotherapy, Chemoradiation

\section{Introduction}

Pancreatic cancer (PC) is one of the most aggressive types of neoplasia. It represents the fourth cause of cancerrelated deaths in the United States [1] and it is projected to become the second leading cause of cancer-related death by 2030 [2]. Nowadays, surgical resection followed by an adjuvant combination of gemcitabine (GEM) and capecitabine has shown the best long-term oncological results and the potential of cure in patients with resectable PC [3]. However, $80 \%-85 \%$ of patients showed unresectable disease at the time of diagnosis due to advanced locoregional or metastatic

Received 26 April 2018, Revised 02 July 2018, Accepted 17 August 2018.

Correspondence: Juan Glinka, MD, Department of Surgery, Hepato-Bilio-Pancreatic Unit, Hospital Italiano de Buenos Aires, Juan D. Perón 4190, C1181 ACH, Buenos Aires, Argentina. Tel: +54-11-4981-4501, E-mail: juan.glinka@hospitalitaliano.org.ar

(c) This is an Open Access article distributed under the terms of the Creative Commons Attribution Non-Commercial License (http://creativecommons.org/ licenses/by-nc/4.0/) which permits unrestricted non-commercial use, distribution, and reproduction in any medium, provided the original work is properly cited.

www.e-roj.org 
pancreatic disease. Although palliative chemotherapy is the treatment of choice in the metastatic setting, the management of the locally advanced disease is still controversial [4]. Locally advanced unresectable pancreatic cancer (LAPC) is defined as an extended involvement of arterial vessels or inferior vena cava as well as a superior mesenteric vein and portal vein involvement without any possibility of surgical reconstruction [5]. These patients frequently develop obstructive jaundice or gastric outlet obstruction, and most of them will require a palliative procedure, either minimally invasive or conventional surgery to relieve symptoms. Several oncological treatment options should be considered and include neoadjuvant therapy with subsequent resection, or palliative radiotherapy and chemotherapy [6]. The benefit of chemotherapy is well described $[4,7]$; however, there is controversy about the use of radiation in patients with LAPC explored for resective purposes and declared surgically unresectable.

Therefore, the purpose of this work is to compare a particular population of patients with a palliative double bypass due to LAPC treated or not with radiotherapy after surgery and its impact on their survival.

\section{Materials and Methods}

\section{Study design and definitions}

We have retrospectively reviewed clinical records of patients with palliative double bypass for LAPC between June 2006 and August 2012. Institutional Review Board gave ethical approval to perform this study (No. 2717). The cohort was divided into two groups: one treated with postoperative radiotherapy vs. other non-exposed group who received standard palliative chemotherapy. Baseline characteristics, postoperative complications related to treatment and their relation to the main event (mortality) were compared.

LAPC was defined according to the consensus statement established by the International Study Group of Pancreatic Surgery [5].

Postoperative complications were evaluated according to the Clavien-Dindo classification [8]. The adverse effects of radiotherapy were categorized according to the Radiation Therapy Oncology Group (RTOG) and the European Organisation for Research and Treatment of Cancer (EORTC) acute toxicity guidelines [9].

Patterns of recurrence after treatment were categorized as locoregional (evidence for disease progression in the tumor bed or regional lymph nodes) or distant metastasis (other organs, peritoneal carcinomatosis or non-regional lymph node metastasis). The diagnosis of recurrence was made through cross-sectional imaging during the follow-up. Overall disease recurrence was also considered.

Follow-up information was obtained by retrospective analysis of medical reports or by direct contact with patients.

\section{Institution protocol}

All patients with a diagnosis of a PC are studied with the multidetector computed tomography scan (MDCT) with triphasic protocol to evaluate resectability. Patients with crosssectional imaging compatible with resectable PC or borderline resectable pancreatic cancer (BRPC), where no preoperative evidence of metastatic disease or local tumor growth excluding potential curative resection is found, are taken to exploratory surgery.

When an intraoperative non-resectability is proven to these patients (intraoperative diagnosis of LAPC), we institutionally offer them a double bypass, and they constitute our population under study.

Patients with LAPC criteria by cross-sectional imaging, were not taken for exploratory surgery and therefore were not considered in the study. In Fig. 1, we summarize our institution management diagram for patients with PC. Endoscopic retrograde cholangiopancreatography (ERCP) and plastic stent placement were selectively performed in patients with obstructive jaundice. In such cases, surgery is carried out in the next 30 days until total bilirubin levels were less than 200 $\mu \mathrm{mol} / \mathrm{L}$ (normal value, 1.7 to $20.5 \mu \mathrm{mol} / \mathrm{L}$ ).

In patients with curative surgical chances, we started with subcostal bilateral laparotomy and make an abdominal exploration to rule out hepatic metastases, peritoneal and omental dissemination or ascites. We did not use routine staging laparoscopy, which is left to the discretion of the operating surgeon. If the tumor was unresectable at the surgical exploration, a gastric and biliary bypass was made. In all cases, we used a Roux-en-Y hepaticojejunostomy (side to side without transecting the bile duct) and we performed a retrocolic gastrojejunostomy in one layer with a handsewn and using a resorbable running suture. Patients whether stented received imipenem $500 \mathrm{mg}$ every 6 hours for perioperative prophylaxis. Patients who were not stented received usual antibiotic prophylaxis with a third generation of cephalosporins. Later in the postoperative period, the adequacy of antibiotics was based on the bile and biliary stent culturesensitivity report, if required.

When the patient was discharged, the studies and planning of the oncological therapy began within the next 2 weeks, as 


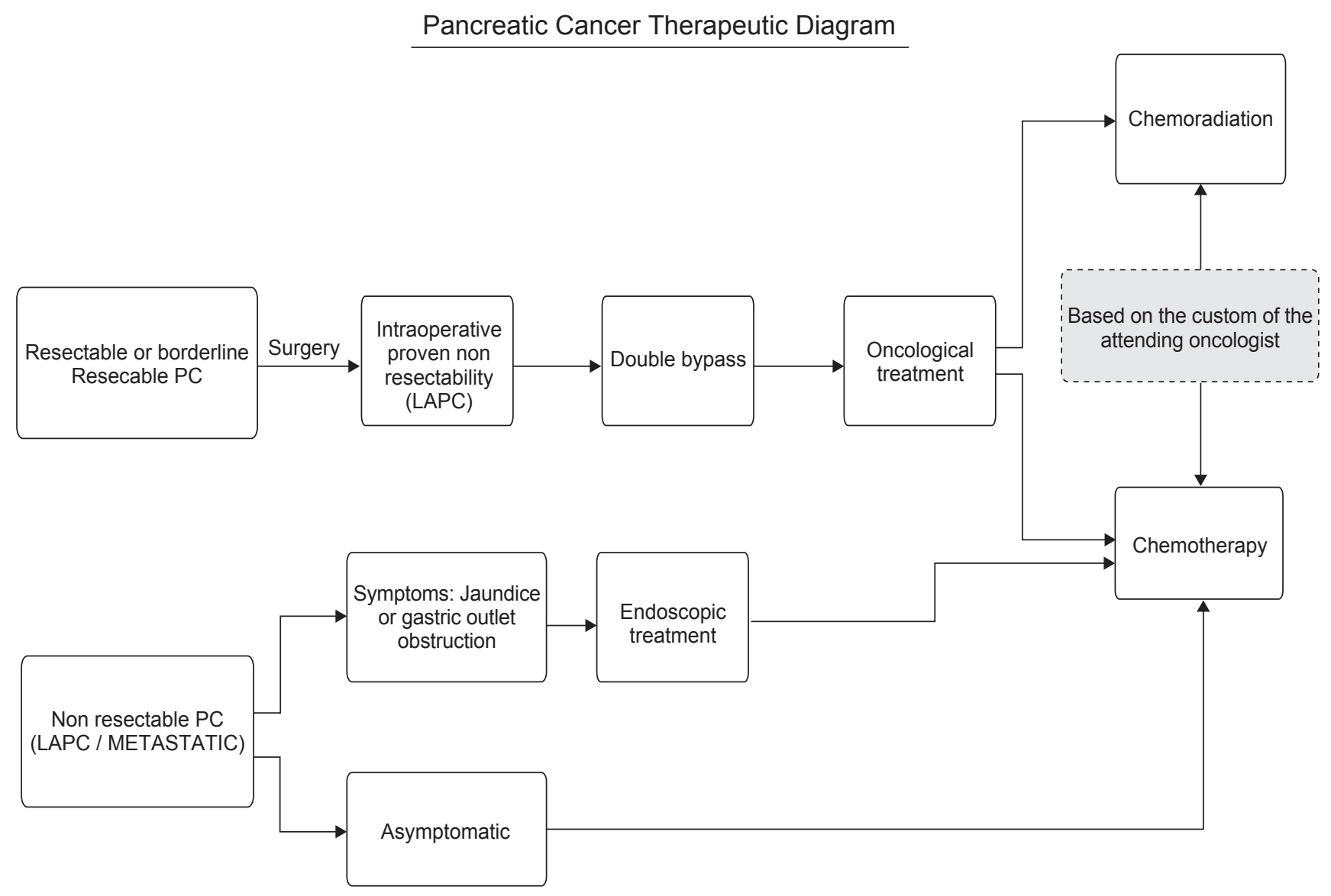

Fig. 1. Pancreatic cancer therapeutic diagram of our institution. PC, pancreatic cancer; LAPC, locally advanced pancreatic cancer.

long as the patient's clinical general status is acceptable.

Regarding the chemotherapy scheme, patients received GEM $\left(1,000 \mathrm{mg} / \mathrm{m}^{2}\right)+$ oxaliplatin $\left(85 \mathrm{mg} / \mathrm{m}^{2}\right)$ every 14 days (between four and six cycles). After that, and if no clinical or imaging evidence of progression of the disease was observed, the consolidative treatment continues with chemoradiation. During the radiotherapy, we used as radiosensitizer low-dose weekly GEM $\left(300 \mathrm{mg} / \mathrm{m}^{2}\right)$ or capecitabine $\left(830 \mathrm{mg} / \mathrm{m}^{2}\right)$ twice a day 5 days a week.

The radiotherapy protocol was implemented based on the criteria of the treating group. Radiotherapy technique included three-dimensional conformal therapy and intensitymodulated radiotherapy, for total doses of 45-50.4 Gy, the fraction was given by $1.8 / 2 \mathrm{~Gy}, 5$ days a week. A planning MDCT was required to define target volumes (Fig. $2 \mathrm{~A}-2 \mathrm{C}$ ). The gross tumor volume (GTV) was determined during the MDCT planning computed tomography scan, portion by portion using the treatment planning system software. The clinical target volume (CTV) was defined as the GTV + expansions: pancreatic area, hepaticojejunostomy, gastrojejunostomy, regional lymph nodes (peripancreatic, celiac, superior mesenteric, porta hepatis, and para-aortic-defined by RTOG contouring atlas consensus).

The two expansions used for the CTV were (1) Expansion 1: pancreatic area, portal vein, celiac trunk, superior mesenteric artery with $1 \mathrm{~cm}$ of isotropic expansion. (2) Expansion 2: anisotropic expansion of the aorta, $2.5 \mathrm{~cm}$ to the right, $1 \mathrm{~cm}$ to the left, $2 \mathrm{~cm}$ anterior wall, and $0.2 \mathrm{~cm}$ posterior wall. While the GTV plus the respective expansions determine the CTV, the planning target volume results from the combination between the CTV plus $0.5 \mathrm{~cm}$ of isotropic expansion.

\section{Statistical analysis}

Categorical variables are described using percentages. Continuous variables are expressed as the mean and standard deviation for those which are symmetrically distributed. And, the mean (interquartile range) for those non-symmetrically distributed. The association between the outcome and the 

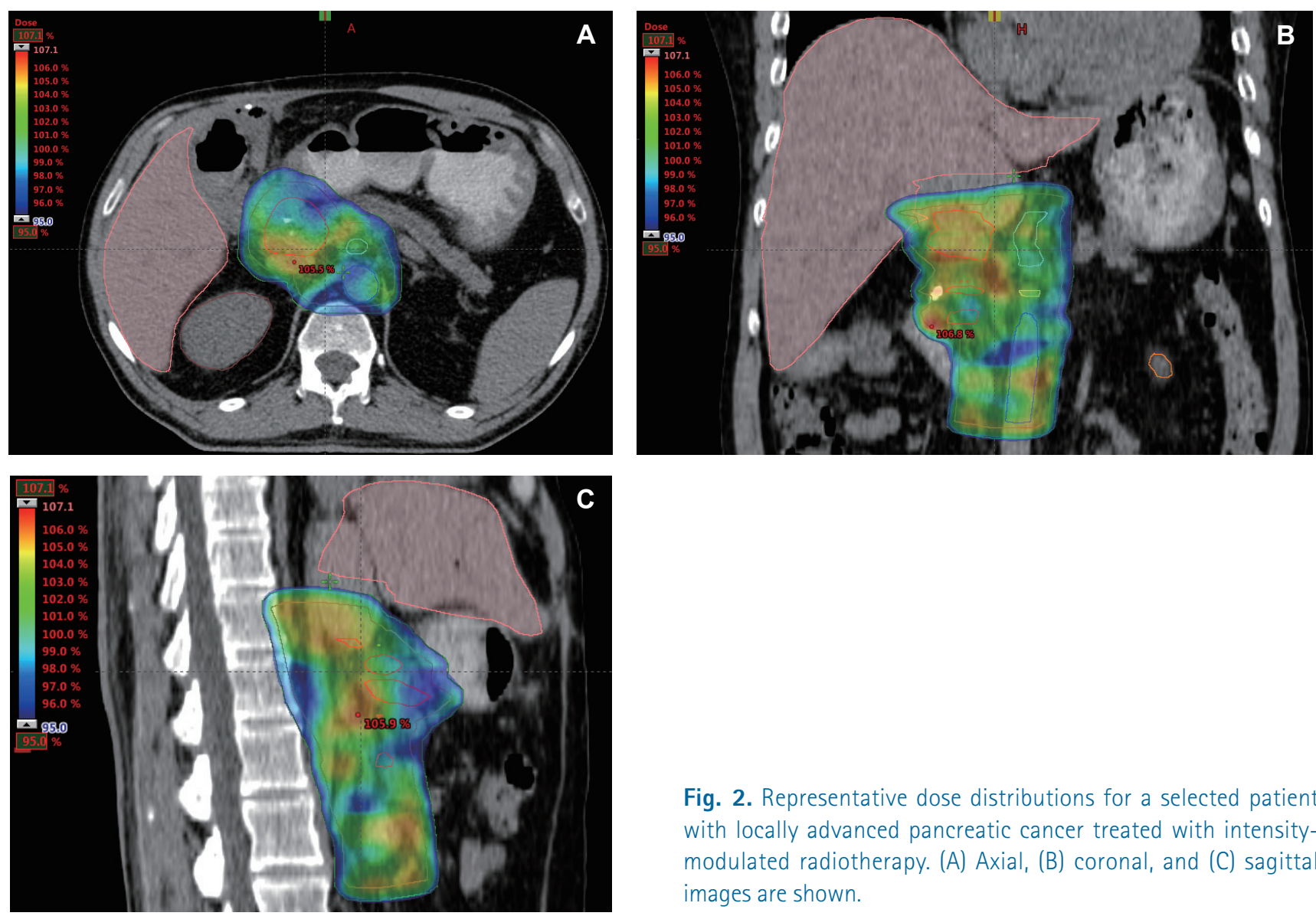

Fig. 2. Representative dose distributions for a selected patient with locally advanced pancreatic cancer treated with intensitymodulated radiotherapy. (A) Axial, (B) coronal, and (C) sagittal images are shown.

treatment was assessed using the chi-square test or MannWithney as appropriate. Survival of 1 and 2 years was analyzed using Kaplan-Meier curves and compared with the log-rank test. Hazard ratio was estimated with the Cox proportional model. Statistically significant probabilities < $5 \%$ were considered. The statistical analysis was carried out by SPSS version 16 software (SPSS Inc., Chicago, IL, USA).

\section{Results}

Seventy-four patients were included and divided in an exposed group ( $n=36$ ) and a non-exposed group $(n=38)$. The mean follow-up was of 12 months (range, 2 to 137 months). Baseline characteristics are described in Table 1.

All patients have been taken up for surgery with the aim of curative resection, but they had an unresectable disease. All patients have histopathological confirmation of pancreatic adenocarcinoma. There were 15 postoperative complications (morbidity 18.9\%) in 14 patients, without 30-day-postoperative mortality. Table 2 summarized the type of postoperative surgical complication and their subsequent treatment. There were no differences between postoperative

Table 1. Baseline characteristics of the study population

\begin{tabular}{lccc}
\hline \multicolumn{1}{c}{ Variable } & Exposed group $(n=36)$ & Non-exposed group $(n=38)$ & $p$-value \\
\hline Sex, male & $23(64)$ & $19(50)$ & 0.22 \\
Age $(y r)$ & $65.5 \pm 0.8$ & $69.0 \pm 9.5$ & 0.10 \\
Karnofsky Performance Status & $70(60-80)$ & $70(60-80)$ & 0.47 \\
Tumor size $(\mathrm{mm})$ & $34(28-51)$ & $39.5(30-46)$ & 0.57 \\
CA 19-9 $(\mathrm{IU} / \mathrm{mL})$ & $1,010(63-5,000)$ & $384(56-1,300)$ & 0.11 \\
\hline
\end{tabular}

Values are presented as number $(\%)$ or mean \pm standard deviation or median (interquartile range). 
Table 2. Surgical complications and their treatment

\begin{tabular}{|c|c|c|c|c|}
\hline Complication & $\begin{array}{l}\text { Exposed } \\
\text { group }\end{array}$ & $\begin{array}{l}\text { Non-exposed } \\
\text { group }\end{array}$ & $\begin{array}{l}\text { Clavien-Dindo } \\
\text { classification }\end{array}$ & Treatment \\
\hline Delayed gastric emptying & 2 & 2 & $\|$ & Medical \\
\hline Cholangitis & 3 & 1 & $\|$ / IIlb & $\begin{array}{l}\text { Medical management and } \\
\text { percutaneous dilatation }\end{array}$ \\
\hline Urinary infection & 1 & 0 & ॥ & Medical \\
\hline Wound infection & 3 & 1 & $\|$ & Medical \\
\hline Intraabdominal abscess & 0 & 1 & Illa & CT guided percutaneous drainage \\
\hline Gastroenteric anastomosis ulcer & 0 & 1 & $\| 11 b$ & $\begin{array}{c}\text { Medical treatment } \\
\text { Endoscopy + hemostasis }\end{array}$ \\
\hline Total complications ${ }^{\text {a) }}$ & $9(25)$ & $6(15.7)$ & - & - \\
\hline
\end{tabular}

Values are presented as number (\%).

$\mathrm{CT}$, computed tomography.

a) $p=0.325$.

complications and the indication of receiving radiotherapy afterwards (complications, 15.7\% in non-exposed group and $25 \%$ in exposed group; $p=0.325$ ).

Regarding radiotherapy, $86 \%$ of patients $(n=31)$ have completed treatment receiving a total dose $>45.6$ Gy and $13.8 \%(n=5)$ did not finish it (receiving full doses of 24-40 Gy). Five patients (13.8\%) did not complete radiotherapy, two due to persistent delayed gastric emptying and regular general condition, and the other three because they were treated in another institution with the consequent loss in their followup. Complications (toxicity) were related to upper and lower gastrointestinal tract in all cases (67.9\% of exposed group). We register 53 complications in 24 patients, although (98.1\%) were mild (grade I and II) (Table 3).

Table 3. Adverse events related to radiotherapy $(n=36)$

\begin{tabular}{lcccc}
\hline \multicolumn{1}{c}{ Gl toxicity } & Grade I & Grade II & Grade III & Grade IV \\
\hline Anorexy & 17 & 4 & 1 & - \\
Diarrhea & 11 & 2 & - & - \\
Nausea/vomiting & 13 & 5 & - & - \\
\hline Gl, gastrointestinal. & & & &
\end{tabular}

Table 4. Patterns of recurrence in both groups

\begin{tabular}{lccc}
\hline \multicolumn{1}{c}{ Recurrence } & Non-exposed group & Exposed group & p-value \\
\hline Locoregional & $31(81.5)$ & $11(30.5)$ & 0.001 \\
Distant & $26(68.4)$ & $33(91.6)$ & 0.013 \\
Overall & $37(97.4)$ & $34(94.4)$ & 0.542 \\
\hline
\end{tabular}

Values are presented as number (\%).
Patterns of recurrence in patients of the exposed group and the non-exposed group are summarized in Table 4.

As observed in the survival curves (Fig. 3) patients of nonexposed group had an overall survival of 1 and 2 years of $50 \%$ and $28 \%$, while $64 \%$ and $35 \%$ for patients with exposed group ( $p=0.11$; power $72 \%$; hazard ratio $=0.53 ; 95$ confidence interval, 0.24-1.18).

\section{Discussion and Conclusion}

The optimal management of patients with LAPC is still controversial, and the therapeutic criteria are not homogeneous in daily practice, neither in the literature nor clinical guidelines.

Systemic chemotherapy is the primary treatment for patients with LAPC or metastatic disease. Numerous clinical trials and meta-analyses have demonstrated the vital role of GEM compared with 5-FU in survival improvement in three clinical settings: neoadjuvant therapy, downsizing of LAPC, and palliative treatment in PC stage IV $[3,7]$. Nowadays, GEM-based chemotherapy is one of the standard treatments for PC.

The basis for incorporating radiotherapy in PC and especially in LAPC is arguable. The first description of a probable benefit 


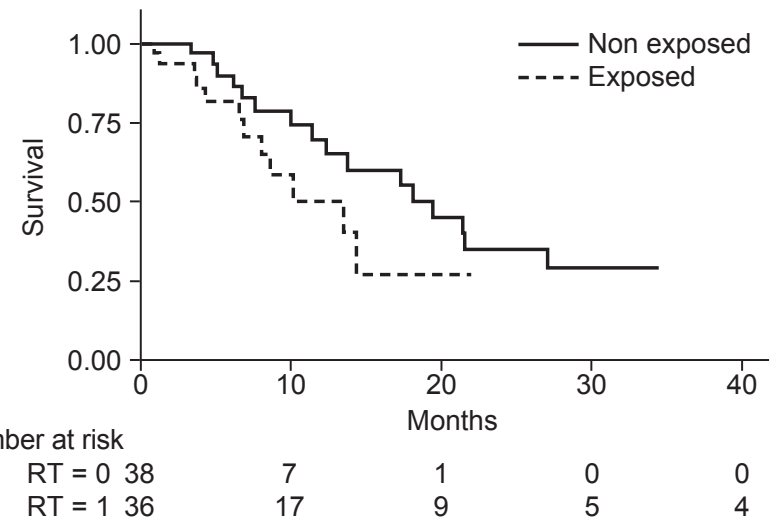

Fig. 3. Survival curves of 36 patients treated with RT (exposed group) and of 38 without RT (non-exposed group). With an overall survival of 1 and 2 years of $50 \%$ and $28 \%$, and $64 \%$ and $35 \%$, respectively. $\mathrm{RT}$, radiotherapy.

in this kind of patients was based on a Mayo Clinic randomized controlled trial (RCT), which showed an improvement in survival with this strategy [10]. However, there have been several opposite opinions about the impact of radiotherapy in the survival of these patients [11]. We must highlight that RCTs in this topic, have used fluoropyrimidine-based chemotherapy and used radiation equipment, dosages and schedules that are not considered adequate by today's standards. Otherwise, these studies were not carried out using GEM as a radiosensitizer $[12,13]$. Therefore, it is difficult to compare the historical evidence with current standards.

The use of radiotherapy has not been generalized due to reports of treatment-related toxicity, with the consequent morbidity and deterioration in the quality of life [14]. Nowadays, advances in the field of radiation oncology, particularly those in radiation treatment delivery techniques, could lead improvements not only in an apparently local tumor control but a reduction in toxicity rates. Now, it is known that the studies that report the highest degrees of toxicity are those related to radiation doses exceeding $60 \mathrm{~Gy}$ [15]. In our series, no patient exceeded doses of 45-50.4 Gy, which entailed a percentage of completion of the treatment of about $86 \%$ with no severe toxicity. Only gastrointestinal complications categorized as grade 1-2 without any hospitalizations has been observed.

Regarding survival improvement of radiotherapy, some trials have been carried out in this particular population. The ECOG4201 [16] trial was designed to evaluate the role of radiation with concurrent GEM compared with GEM alone in patients with LAPC. They observed that concomitant treatment prolonged the survival (11 months vs. 9.2 months) and yielded more than 1-year survival rate (50\% vs. $32 \%$ ), without significant adverse effects on quality of life or severe toxicities. The GERCOR study [17] demonstrated that patients with LAPC who received chemoradiotherapy, after three months of chemotherapy with GEM and oxaliplatin (GEMOX), had significantly better survival rates than those who continued with chemotherapy alone. The critical point is that the strategy based on the induction chemotherapy can select a subgroup of patients (up to 70\%) without early progression who may potentially benefit from chemoradiotherapy. The other subset of patients (approximately 30\%) have an occult metastatic disease at diagnosis and, thus, they will undoubtedly not benefit from administering chemoradiotherapy as firstline treatment, and they should continue only with systemic therapy. This was also the primary strategy for the treatment of the patients of our series that underwent radiotherapy. Recently the LAP07 trial [18] included patients with LAPC controlled after 4 months of induction chemotherapy with GEM, without a significant difference in overall survival with chemoradiotherapy compared with GEM-based chemotherapy scheme alone as a definitive treatment. Moreover, patients who underwent surgery were only $4 \%$ with only $2.5 \%$ of Ro resection.

While there is still no substantial evidence about the use of radiotherapy, in our series the combination of GEMOX and radiation showed a tendency towards a longer overall survival compared with the chemotherapy-only group ( 1 and 2 years survival $64 \%$ and $35 \%$ vs. $50 \%$ and $28 \%$, respectively).

Analyzing the patterns of recurrence in both groups, we observed a significant difference in locoregional disease control in favor of the irradiated group. However, we also found a significant difference in which this cohort also developed more distant recurrence. A possible explanation for this striking phenomenon is that, although radiotherapy would be useful in locoregional control, it would be accompanied by a low dose of systemic chemotherapy during its administration. Therefore, with this combination, distance dissemination would be favored as stated in the ESPAC-1 trial [19]. Consequently, we believe that improvements in systemic treatment during radiation could improve its usefulness with less distant recurrence. In other words, it would be necessary to find a systemic therapy that allows obtaining an optimal systemic control and with acceptable toxicity to administer it together with the radiation treatment.

Nowadays, the landscape for systemic therapy for PC has 
changed significantly since the PRODIGE 4/ACCORD 11 RCT [20]. The use of Folfirinox, a combination of 5-FU, oxaliplatin, irinotecan, and leucovorin has demonstrated superiority over GEM monotherapy in patients with metastatic PC. A response rate of $31.6 \%$ vs. $9.4 \%$ and mean overall survival of 11.1 months vs. 6.8 months $(p=0.001)$ has been reported. No RCT has been performed with FOLFIRINOX for LAPC patients. Nevertheless, in Rombouts et al. [21], systematic review on clinical outcomes after FOLFIRINOX-based treatment for LAPC demonstrated apparently a real downstaging with a $28 \%$ resection rate (77\% were $\mathrm{R} 0$ ) and a mean overall survival ranging between 8.9 and 25.0 months, in which approximately $50 \%$ of patients received radiotherapy. Hackert et al. [22] demonstrated $60 \%$ of resectability with neoadjuvant FOLFIRINOX in LAPC with no radiotherapy at all, compared to a GEM + radiotherapy group. In this scenario of FOLFIRINOX therapy, the concomitant role of radiation to systemic treatment, is still unknown $[23,24]$.

The use of double bypass surgery is not universally accepted to manage locally advanced tumors, but we usually reserve it as our best and final option for patients declared unresectable intraoperatively and with no evidence of disseminated disease, in which a more prolonged survival is expected $[25,26]$. This study has as strength the homogeneity of the study population because both groups are comparable cohorts: all suitable for surgery and without significant demographic differences. The only difference between them was the addition of radiotherapy in a selected group based on clinical expertise.

Concerning surgical results, we have reported a globally low rate of complications (18.9\%) and mostly mild, such as the tendency observed by Kneuertz et al. [27] for this type of surgeries, and, therefore, allowed early access to oncological treatment.

Even though we tended to achieve a survival improvement in the irradiated cohort, we do not re-explore with resective intentions any non-progressive patients of the study, conditioned by the limited evidence of such practice at the time of the investigation.

The study also has some limitations. The retrospective design and relatively small population under study have not enough power to demonstrate the significant benefits of postoperative radiotherapy in patient survival. Whence it is imperative to develop randomized and prospective trials to guide more specific treatment lines in this particular population.

In conclusion, the optimal management of patients with LAPC is still controversial, and the therapeutic criteria are not homogeneous in daily practice, neither in the literature nor clinical guidelines. Chemotherapy is the standard of care in patients with LAPC, and the addition of concomitant radiotherapy might improve patients' survival, without severe morbidity. However, given the more significant systemic progression in the irradiated population, improvements in systemic treatment during its administration could increase its usefulness.

\section{Conflict of Interest}

No potential conflict of interest relevant to this article was reported.

\section{References}

1. Fitzgerald TL, Hickner ZJ, Schmitz M, Kort EJ. Changing incidence of pancreatic neoplasms: a 16-year review of statewide tumor registry. Pancreas 2008;37:134-8.

2. Rahib L, Smith BD, Aizenberg R, Rosenzweig AB, Fleshman JM, Matrisian LM. Projecting cancer incidence and deaths to 2030: the unexpected burden of thyroid, liver, and pancreas cancers in the United States. Cancer Res 2014;74:2913-21.

3. Neoptolemos JP, Palmer DH, Ghaneh P, et al. Comparison of adjuvant gemcitabine and capecitabine with gemcitabine monotherapy in patients with resected pancreatic cancer (ESPAC-4): a multicentre, open-label, randomised, phase 3 trial. Lancet 2017;389:1011-24.

4. Balaban EP, Mangu PB, Khorana AA, et al. Locally advanced, unresectable pancreatic cancer: American Society of Clinical Oncology Clinical Practice Guideline. J Clin Oncol 2016;34:2654-68.

5. Bockhorn M, Uzunoglu FG, Adham M, et al. Borderline resectable pancreatic cancer: a consensus statement by the International Study Group of Pancreatic Surgery (ISGPS). Surgery 2014;155:977-88.

6. Werner J, Combs SE, Springfeld C, Hartwig W, Hackert T, Buchler MW. Advanced-stage pancreatic cancer: therapy options. Nat Rev Clin Oncol 2013;10:323-33.

7. Burris HA 3rd, Moore MJ, Andersen J, et al. Improvements in survival and clinical benefit with gemcitabine as firstline therapy for patients with advanced pancreas cancer: a randomized trial. J Clin Oncol 1997;15:2403-13.

8. Clavien PA, Barkun J, de Oliveira ML, et al. The Clavien-Dindo classification of surgical complications: five-year experience. Ann Surg 2009;250:187-96.

9. Cox JD, Stetz J, Pajak TF. Toxicity criteria of the Radiation Therapy Oncology Group (RTOG) and the European 
Organization for Research and Treatment of Cancer (EORTC). Int J Radiat Oncol Biol Phys 1995;31:1341-6.

10. Moertel CG, Childs DS Jr, Reitemeier RJ, Colby MY Jr, Holbrook MA. Combined 5-fluorouracil and supervoltage radiation therapy of locally unresectable gastrointestinal cancer. Lancet 1969;2:865-7.

11. Sultana A, Tudur Smith $C$, Cunningham D, et al. Systematic review, including meta-analyses, on the management of locally advanced pancreatic cancer using radiation/combined modality therapy. Br J Cancer 2007;96:1183-90.

12. Habermehl D, Kessel K, Welzel T, et al. Neoadjuvant chemoradiation with Gemcitabine for locally advanced pancreatic cancer. Radiat Oncol 2012;7:28.

13. Goel N, Reddy SS. Randomized clinical trials in pancreatic cancer. Surg Oncol Clin N Am 2017;26:767-90.

14. Zhang $X$, Huang HJ, Feng D, Yang DJ, Wang CM, Cai QP. Is concomitant radiotherapy necessary with gemcitabine-based chemotherapy in pancreatic cancer? World J Gastroenterol 2014;20:17648-55.

15. Chauffert B, Mornex F, Bonnetain F, et al. Phase III trial comparing intensive induction chemoradiotherapy (60 Gy, infusional 5-FU and intermittent cisplatin) followed by maintenance gemcitabine with gemcitabine alone for locally advanced unresectable pancreatic cancer. Definitive results of the 2000-01 FFCD/SFRO study. Ann Oncol 2008;19:1592-9.

16. Loehrer PJ Sr, Feng Y, Cardenes $H$, et al. Gemcitabine alone versus gemcitabine plus radiotherapy in patients with locally advanced pancreatic cancer: an Eastern Cooperative Oncology Group trial. J Clin Oncol 2011;29:4105-12.

17. Huguet F, Andre T, Hammel P, et al. Impact of chemoradiotherapy after disease control with chemotherapy in locally advanced pancreatic adenocarcinoma in GERCOR phase II and III studies. J Clin Oncol 2007;25:326-31.

18. Hammel $P$, Huguet $F$, van Laethem $J L$, et al. Effect of chemoradiotherapy vs chemotherapy on survival in patients with locally advanced pancreatic cancer controlled after 4 months of gemcitabine with or without erlotinib: the LAP07 randomized clinical trial. JAMA 2016;315:1844-53.

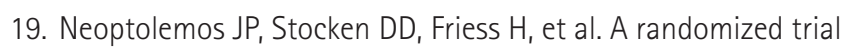
of chemoradiotherapy and chemotherapy after resection of pancreatic cancer. N Engl J Med 2004;350:1200-10.

20. Gourgou-Bourgade S, Bascoul-Mollevi C, Desseigne F, et al. Impact of FOLFIRINOX compared with gemcitabine on quality of life in patients with metastatic pancreatic cancer: results from the PRODIGE 4/ACCORD 11 randomized trial. J Clin Oncol 2013;31:23-9.

21. Rombouts SJ, Walma MS, Vogel JA, et al. Systematic review of resection rates and clinical outcomes after FOLFIRINOX-based treatment in patients with locally advanced pancreatic cancer. Ann Surg Oncol 2016;23:4352-60.

22. Hackert $T$, Sachsenmaier M, Hinz U, et al. Locally advanced pancreatic cancer: neoadjuvant therapy with FOLFIRINOX results in resectability in $60 \%$ of the patients. Ann Surg 2016;264:457-63.

23. Ferrone CR, Marchegiani G, Hong TS, et al. Radiological and surgical implications of neoadjuvant treatment with FOLFIRINOX for locally advanced and borderline resectable pancreatic cancer. Ann Surg 2015;261:12-7.

24. Kim SS, Nakakura EK, Wang ZJ, et al. Preoperative FOLFIRINOX for borderline resectable pancreatic cancer: is radiation necessary in the modern era of chemotherapy? J Surg Oncol 2016;114:587-96.

25. Scott EN, Garcea G, Doucas H, Steward WP, Dennison $A R$, Berry DP. Surgical bypass vs. endoscopic stenting for pancreatic ductal adenocarcinoma. HPB (Oxford) 2009;11:11824.

26. Koninger J, Wente MN, Muller MW, Gutt CN, Friess H, Buchler MW. Surgical palliation in patients with pancreatic cancer. Langenbecks Arch Surg 2007;392:13-21.

27. Kneuertz PJ, Cunningham SC, Cameron JL, et al. Palliative surgical management of patients with unresectable pancreatic adenocarcinoma: trends and lessons learned from a large, single institution experience. J Gastrointest Surg 2011;15:1917-27. 